\title{
Investigation of the protective effect of erythropoietin on spinal cord injury in rats
}

\author{
ZHENGHUA HONG, HUAXING HONG, HAIXIAO CHEN, ZHANGFU WANG and DUN HONG \\ Department of Orthopedics, Taizhou Hospital of Zhejiang Province, Taizhou 317000, P.R. China
}

Received May 2, 2011; Accepted June 6, 2011

DOI: 10.3892/etm.2011.285

\begin{abstract}
Erythropoietin (EPO) is a promising therapeutic agent used in a variety of spinal cord injuries. Therefore, identifying the specific molecular pathway mediating the neuronal protective effect of EPO after spinal cord injury (SCI) is of great value to the patients concerned. Platelet-derived growth factor (PDGF)-B is an important factor in the recovery of neurological function. We explored changes in the expression of PDGF-B in spinal cord injury rats after receiving EPO treatment. We used a weight-drop contusion SCI model, and EPO treatment group rats received single doses ofEPO (1,000 U/ $\mathrm{kg}$ i.p.) immediately after the operation. Seven days after the operation, the results revealed a more rapid recovery as noted by the higher BBB scores, less disruption and more neuronal regeneration of the spinal cord in the EPO treatment group than that in the SCI group. PDGF-B expression also increased in the EPO treatment group compared to that in the SCI group $(\mathrm{P}<0.01)$. This study showed that PDGF-B plays a role in the neuronal protective effect of EPO on spinal cord injury in rats, which may help to explain the quick recovery after EPO treatment of spinal cord injury.
\end{abstract}

\section{Introduction}

Spinal cord injury (SCI) is a severe worldwide health problem resulting from different etiological factors such as traffic accidents. SCI can be divided into two stages: the acute phase which is caused by directly tissue compression and hemorrhage, and the secondary injury phase, in which secondary

Correspondence to: Dr Haixiao Chen, Department of Orthopedics, Taizhou Hospital of Zhejiang Province, 150 Ximen Street, Taizhou 317000, P.R. China

E-mail: hongzhenghua2008@163.com

Abbreviations: EPO, erythropoietin; SCI, spinal cord injury; PDGF-B, platelet-derived growth factor-B; CNS, central nervous system; SD rat, Sprague-Dawley rat; BBB scale, Basso, Beattie and Bresnahan locomotor rating scale

Key words: platelet-derived growth factor-B, spinal cord injury, erythropoietin, rat cell apoptosis occurs at the lesion site, affecting both neurons and astrocytes $(1,2)$ It has long been believed that the central nervous system (CNS) is incapable of regenerating itself after injury and during the repair process after SCI. Thus, an ideal treatment would be one administered systemically but without significant side effects. Erythropoietin (EPO), a potent inhibitor of apoptosis and a promising therapeutic agent, has been used in a variety of neurological insults, including traumatic brain injury, brain stroke and spinal cord injury $(3,4)$. Therefore, identifying the specific molecular pathway mediating the EPO neuronal protective effects of EPO after SCI is of great value to the patients concerned.

Platelet-derived growth factor (PDGF) is synthesized and secreted by multiple types of cells and can bind to its specific receptor (PDGFR) in the CNS (5). Due to the reported neurotrophic and neuroprotective effects of exogenously administered PDGFs. PDGFs are speculated to play a role in CNS development, maintenance, and response to CNS injury (5). The PDGF family has four gene products (PDGF-A-D) that act via two classical receptor tyrosine kinases, PDGF- $\alpha \mathrm{R}$ and PDGF- $\beta$ R. Members of the PDGF family have multiple roles during embryogenesis and in a variety of pathological situations in the adult (6). In addition, PDGFs can maintain the reactivity of the neuron and facilitate the growth of the axon (7). PDGF-B, a member of the PDGF family, has the capacity to promote the mitosis of glial cells and exhibits a neuroprotective effect in the rat cerebrum both during development and in the mature stage. These results indicate the neuroprotective effects of PDGF-B in the function of both the developing and developed brain. Recently scientists have found that EPO accelerates smooth muscle cell-rich vascular lesion formation in mice through endothelial cell activation involving enhanced PDGF release (8). Thus, our working hypothesis is that PDGF-B levels change in spinal cord injury rats after EPO treatment. This study may pave the way for the future treatment of SCI patients.

\section{Materials and methods}

Animal model induction and locomotive function exam. Adult male Sprague-Dawley (SD) rats, weighing 180-200 g, were purchased from the Experimental Animal Centre of Zhejiang University (Hangzhou, China). Rabbit anti-rat PDGF-B antibody, biotinylated goat anti-rabbit secondary antibody and avidin-HRP complex were purchased from Boster Co. (Wuhan, China). 3,3'-Diaminobenzidine hydrochloride was from 
Sigma Co. (St. Louis, MO, USA). Recombinant human EPO was purchased from the China Shenyang Sunshine Pharmaceutical Company Limited (Shenyang, China). All experiments and procedures were approved by the Committee on Animal Care and Use of Zhejiang University. Protocol regarding the animal experiments was approved by the Ethics Committee of the School of Medicine, Zhejiang University, in accordance with NIH guidelines for the ethical care of experimental animals.

Thirty adult male SD rats were randomly divided into three groups (n=10): i) sham operation control group, ii) SCI group and iii) EPO treatment SCI group. Under sodium pentobarbital $(40 \mathrm{mg} / \mathrm{kg}$, i.p.) anesthesia, the vertebral column of the rats was exposed, and a laminectomy was carried out at the T10 level. A contusion injury was then performed using a weight-drop device in the SCI group and EPO treatment SCI group rats. A weight of $10 \mathrm{~g}$ was dropped from a height of $50 \mathrm{~mm}$ on the exposed spinal cord, and the impounder was left for $20 \mathrm{sec}$ before being withdrawn to produce a moderate contusion. EPO treatment SCI group rats received single doses of EPO (1,000 U/kg i.p.) immediately after the incision was closed while the SCI animals received normal saline (via i.p. injection). Sham operation control group animals received the same surgical procedure but sustained no impact injury, and their spinal cord was left exposed for $5 \mathrm{~min}$. They received normal saline (via i.p. injection) immediately after the incision was closed. Seven days after the surgery, rats were laid on the floor and observed for their crawling ability for $5 \mathrm{~min}$. Basso, Beattie and Bresnahan locomotor rating scale (BBB) was used for the evaluation of their locomotive ability.

Immunohistochemical assay and hematoxylin and eosin $(H \& E)$ staining. Seven days after the surgery, five rats in each group were anesthetized with a lethal dose of Nembutal. Their thoracic cavities were opened and perfused intracardially with normal saline. Following saline perfusion, the animals were perfused with $300-400 \mathrm{ml}$ fixative containing $4 \%$ paraformaldehyde in 0.1 M PBS (pH 7.4). After perfusion, the T6-14 segment of the spinal cord of each rat was extracted. The tissues were fixed in the same fixative for $4 \mathrm{~h}$ and then placed in $30 \%$ phosphate-buffered sucrose until the tissue sank. Tissues were routinely processed for paraffin embedding and mounted onto $0.02 \%$ poly-L-lysine-coated slides. The avidin-biotin-peroxidase complex system was used with 3,3'-diaminobenzidine hydrochloride (DAB) as the chromagen. Briefly, tissue sections were washed in PBS, incubated in $1 \%$ bovine serum albumin (BSA) for $30 \mathrm{~min}$, and then incubated overnight at $4^{\circ} \mathrm{C}$ in the primary antibody (1:100 of PDGF-B primary antibody) plus $1 \%$ BSA in PBS. The control sections were incubated in PBS. The next day, the sections were incubated in a biotinylated goat anti-rabbit secondary antibody (diluted to 1:200 in PBS), and subsequently in an avidin-horseradish peroxidase (HRP) solution. Immunolabelling was visualized with $0.05 \mathrm{DAB}$ plus $0.3 \%$ hydrogen peroxide in PBS. The sections were then dehydrated through ethanol and xylene before mounting under coverslips with Permount ${ }^{\mathrm{TM}}$. H\&E staining was also applied to determine the pathological changes in the three groups.

Real-time qPCR assay of the PDGF-B mRNA expression. Seven days after the surgery, the remaining five animals per group were anesthetized with a lethal dose of Nembutal. The
T6-14 segment of the spinal cord of each rat was extracted immediately. For real-time RT-qPCR analysis, total-RNA was isolated from the spinal cord segment using the TRIzol ${ }^{\circledR}$ reagent kit according to the manufacturer's instructions. The RNA concentration was measured spectrophotometrically, and $2 \mu \mathrm{g}$ of total-RNA was added to the complementary DNA synthesis reaction system $(20 \mu \mathrm{l})$. The reaction mixture comprised $4 \mu \mathrm{l}$ of 5X RT buffer, $2.5 \mu \mathrm{mol} / 1$ oligodeoxythymidylic acid oligo(dt), $5 \mathrm{mmol} / \mathrm{l}$ deoxyribonucleotide triphosphate (dNTP) and $20 \mathrm{U}$ of RNase inhibitor. The hexamers were annealed by incubating the samples to $70^{\circ} \mathrm{C}$ for $5 \mathrm{~min}$. M-MLV reverse transcriptase $(200 \mathrm{U})$ was added, and the mixture was incubated at $42^{\circ} \mathrm{C}$ for $60 \mathrm{~min}$. The reaction was stopped by heating to $72^{\circ} \mathrm{C}$ for $10 \mathrm{~min}$. For real-time RT-qPCR, the reaction mixture $(40 \mu \mathrm{l})$ consisted of $4 \mu \mathrm{l}$ complementary DNA, $35.2 \mu \mathrm{l} \mathrm{SYBR}{ }^{\circledR}$-Green qPCR mix, $0.5 \mu \mathrm{l}$ of $5 \mathrm{U}$ TaqDNA polymerase and $0.3 \mu \mathrm{l}$ of $20 \mathrm{pmol} / \mu 1$ PDGF primer. The complementary DNA was denatured by heating to $94^{\circ} \mathrm{C}$ for $3 \mathrm{~min}$. The template was amplified by 40 rounds of PCR (denaturation at $94^{\circ} \mathrm{C}$ for $10 \mathrm{sec}$, annealing at $60^{\circ} \mathrm{C}$ for $30 \mathrm{sec}$, extension at $72^{\circ} \mathrm{C}$ for $30 \mathrm{sec}$ ) before collecting the fluorescence at $72^{\circ} \mathrm{C}$. Meanwhile, primers were used for the housekeeping gene glyceraldehyde-3-phosphate dehydrogenase (GAPDH) in the real-time RT-qPCR to amplify GAPDH as an internal control for quantifying PDGF-B expression. The primers for PDGF-B were as follows: forward, 5-CTCCATCC GCTCCTTTGATGACCTT-3 and reverse, 5-CAGCTCAGC CCCATCTTCGTCTA-3. The primers for GAPDH were as follows: forward, 5-GGTGGACCTCATGGCCTACAT-3 and reverse, 5-GCCTCTCTCTTGCTCTCAGTATCCT-3. The PCR product length of PDGF-B was 73 base pairs (bp).

Statistical analysis. Slides were examined at x400 magnification and analyzed with UTHSCSA Image Tool 3.0 (University of Texas Medical School at San Antonio, TX, USA). The number of PDGF-B-positive cells was measured. The mean \pm SD for all data was calculated. Statistical analysis was performed using SPSS $^{\circledast}$ version 12.0 statistical software for Windows ${ }^{\circledR}$. The significance of any differences between the three groups was evaluated using the $\mathrm{t}$-test. P-values were two tailed, and a $\mathrm{P}<0.05$ was considered statistically significant. Relative quantitation was carried out using the comparative $\mathrm{C}_{\mathrm{T}}$ method. The $\Delta \mathrm{C}_{\mathrm{T}}$ value was determined by subtracting the average GAPDH $\mathrm{C}_{\mathrm{T}}$ value from the average PDGF $\mathrm{C}_{\mathrm{T}}$ value. The calculation of $\Delta \Delta \mathrm{C}_{\mathrm{T}}$ involves diabetic or EPO treatment group $=\Delta \mathrm{C}_{\mathrm{T}}$ control $-\Delta \mathrm{C}_{\mathrm{T}}$. The PDGF mRNA level in the SCI and $\mathrm{EPO}$ treatment SCI group relative to the control was determined using the formula: $2^{-(\Delta \Delta C T)}$. The PDGF mRNA level in the sham operation group was set to 1 .

\section{Results}

$B B B$ scales and $H \& E$ staining. The sham operation control group animals walked normally after recovery from the anesthesia while rats in the SCI and EPO treatment SCI group exhibited dramatic and bilateral hind limb paralysis with no movement or only slight movements of a joint after injury. The animals were followed up for 7 days post-surgery to score their locomotive activity according to the BBB scale. The locomotive dysfunction was found reproducible, and the BBB score in the SCI rats recovered during 7 days to a plateau below the 
A

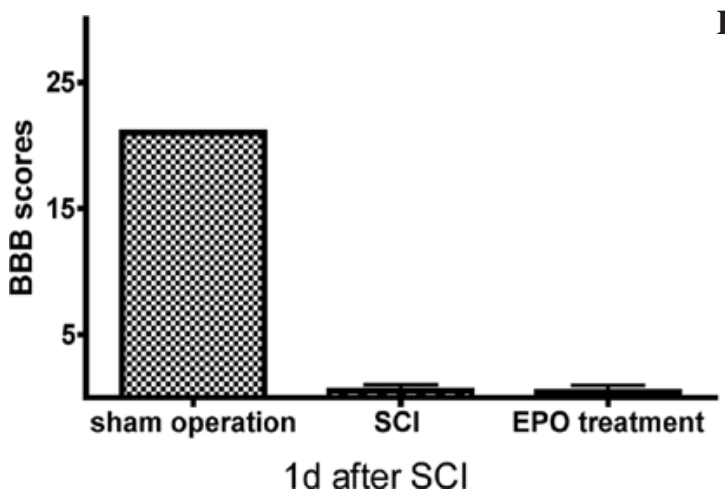

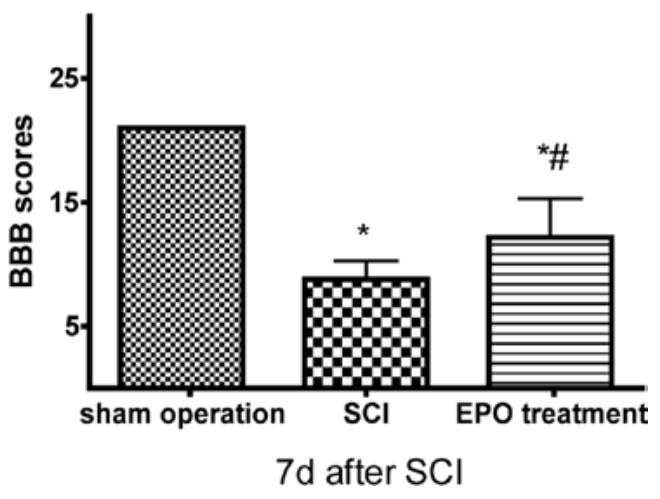

Figure 1. BBB scores in a sham operation control rat, a spinal cord injury rat and an EPO treatment rat 1 (A) and 7 (B) days after SCI. "P $<0.01$, vs. sham operation group; ${ }^{"} \mathrm{P}<0.01$, vs. SCI group.
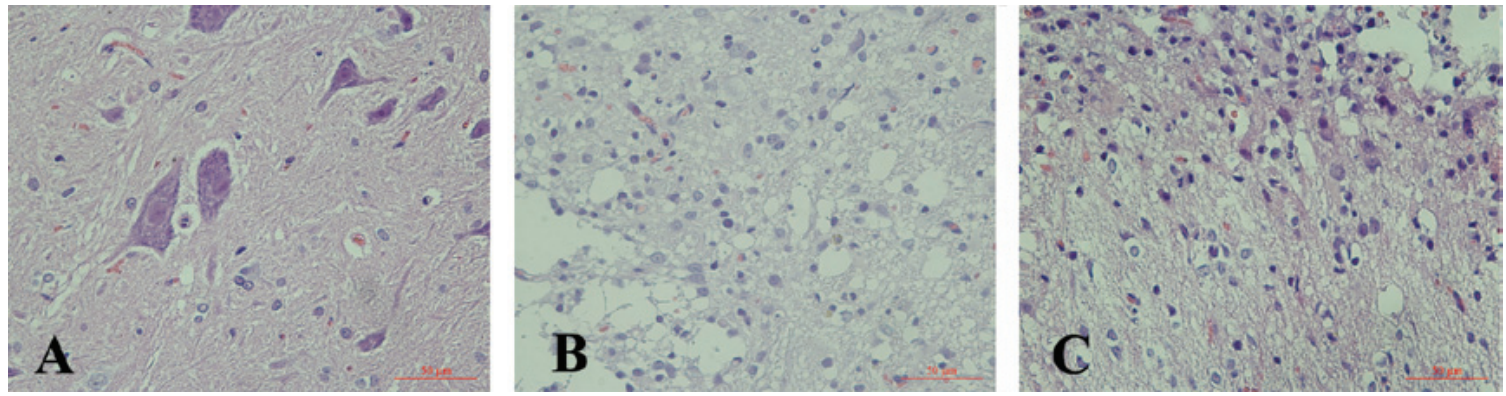

Figure 2. Light photomicrographs of the spinal cord with H\&E staining (x400). (A) A sham operation control rat. (B) A spinal cord injury rat. (C) An EPO treatment rat.
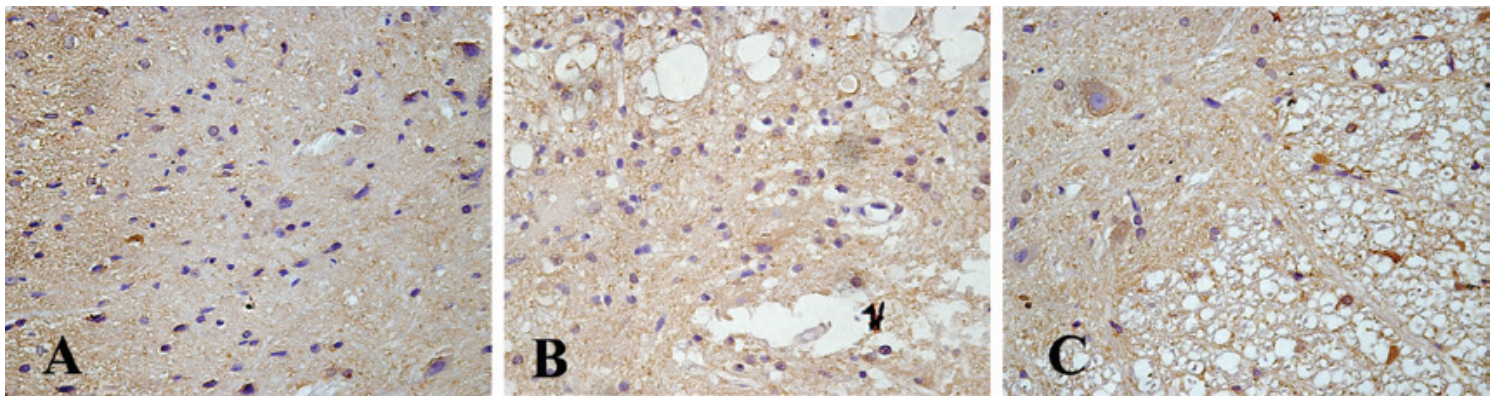

Figure 3. PDGF-B immunohistochemical staining of the spinal cord with DAB staining (x400). (A) A sham operation control rat. (B) A spinal cord injury rat. (C) An EPO treatment rat.

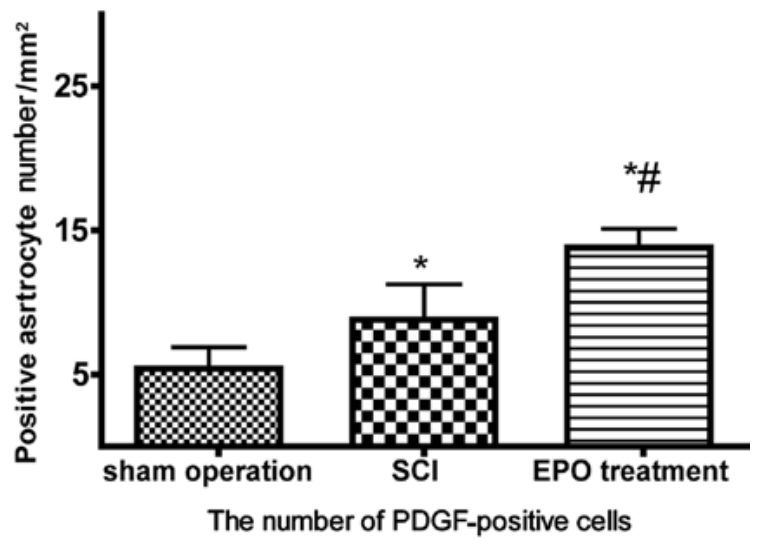

Figure 4. The number of PDGF-B-positive cells in the spinal cord from each group of rats. ${ }^{*} \mathrm{P}<0.01$ vs. operation group; ${ }^{\#} \mathrm{P}<0.01$ vs. SCI group range of 10 points while EPO treatment SCI rats scored above 12 points. This indicated that EPO helped to moderate the severity of the injury and facilitated fast recovered after spinal cord injury $(\mathrm{P}<0.01)$ (Fig. 1).

Consistently, H\&E staining of the lesioned thoracic spinal cord revealed the formation of a large cavity involving the lateral funiculi, dorsal and central gray matter. Seven days after injury we noted some neuronal apoptosis and the numbers of gliocytes increased. A progressive disruption of the dorsal white and central gray matter tissue architecture was noted and gliocytes filled the injury area. Compared with the SCI group, less neuronal degenerating was noted and more gliocytes were observed to fill the injured spinal cord of the EPO treatment SCI rats. The structure of the T10 segment in the sham operation control group was intact and microscopic 
Table I. Relative quantification of PDGF mRNA levels.

\begin{tabular}{lccccc}
\hline Group & $\mathrm{C}_{\mathrm{T}}$ PDGF & $\mathrm{C}_{\mathrm{T}}$ GAPDH & $\Delta \mathrm{C}_{\mathrm{T}}$ & $\Delta \Delta \mathrm{C}_{\mathrm{T}}$ & Fold difference \\
\hline Sham operation & 25.73 & 21.76 & 3.97 & 0 & 1 \\
Spinal cord injury & 23.14 & 20.99 & 2.15 & -1.82 & 3.53 \\
EPO treatment & 22.31 & 20.56 & 1.75 & -2.22 & 4.65 \\
\hline
\end{tabular}

$\Delta \mathrm{C}_{\mathrm{T}}=\mathrm{C}_{\mathrm{T}}$ PDGF $-\mathrm{C}_{\mathrm{T}}$ GAPDH. $\Delta \Delta \mathrm{C}_{\mathrm{T}}=\Delta \mathrm{C}_{\mathrm{T}}^{\text {control }}-\Delta \mathrm{C}_{\mathrm{T}}$. Fold difference $=2^{-(\Delta \Delta \mathrm{CT})}$

structures such as neurons and astrocytes in the grey matter were clear (Fig. 2).

Immunohistochemistry and RT-qPCR assays of the expression of PDGF-B. Cells that stained positive for PDGF-B showed buff-colored granules with DAB staining. There were few PDGF-B-positive cells in the sham operation control group. However, 7 days after SCI, further migration of PDGF-Bpositive cells occurred, and the size of the spinal cord porosis resulting from the trauma was reduced in the EPO treatment SCI group. The PDGF-B-positive cell number was significantly higher in the EPO group compared to that of the SCI group $(\mathrm{P}<0.05)$ (Figs. 3 and 4$)$.

Similar results were noted in the real-time RT-qPCR assay. The PDGF-B mRNA level was up-regulated in the EPO treatment SCI group compared to the SCI group, as indicated by the decreased $\Delta C_{T}$ values (comparative $C_{T}$ method). Specifically, the PDGF-B mRNA level in the SCI group was 3.53 times that of the control group, and this value in the EPO treatment SCI group was 4.65 times that of the control group (Table I).

\section{Discussion}

EPO exhibits neuroprotective effects in a variety of models of central and peripheral nervous system injury $(9,10)$. The local EPO and EPO-R system is markedly engaged in the early stages after compressive spinal cord injury. The reduction in EPO immune-expression and the increase in EPO-R staining strongly support the possible usefulness of a therapeutic approach based on exogenous EPO administration (11). Moreover, EPO may influence the release of neurotransmitters, playing an important role in synaptic plasticity in the adult brain (12). EPO was also found to increase Schwann cell infiltration into the lesion site, although neither compound had any effect on macrophage infiltration either within the lesion site itself or in the surrounding intact tissue (13). Recently, scientists revealed that the binding of EPO to its receptor induces the activation of JAK2, leading to phosphorylation of the inhibitor of nuclear factor- $\kappa \mathrm{B}$. The functional analysis of different receptor forms revealed a correlation of the abilities to induce NF- $\mathrm{B}$ activity and to generate anti-apoptotic signals (14). Zhang et al found that a high dose of EPO attenuates the increase in iNOS expression in the facial nucleus after facial nerve transection and thus may enhance the survival of facial motor neurons (15). The present results showed a more rapid recovery as indicated by higher BBB scores, less disruption and more neuronal regeneration of the spinal cord in the EPO treatment SCI group than that in the SCI group.
This demonstrated that EPO helps to improve the function and pathological changes after SCI in rats.

Members of the PDGF family have multiple roles during embryogenesis and in a variety of pathological situations in the adult. PDGF-B is compatible with normal CNS development and astroglial response to injury (16). To our knowledge, exogenous PDGF was neuroprotective against toxicity induced by HIV-1 Tat in primary midbrain neurons. Administration of PDGF was able to rescue the dopaminergic neurons in the substantia nigra from Tat-induced neurotoxicity. EPO was found to induce expression of PDGF-B, which was at least partially responsible for the induction of Stat5 phosphorylation in SMCs by HUVEC-conditioned medium (8). However, in this study, the expression of PDGF-B in the EPO treatment group was constantly high, exhibiting rapid ability to repair the injured tissue and regain neurological function.

After SCI, the PDGF-positive cell numbers were found to be significantly higher in the spinal cord $(17,18)$. Recent studies indicate that PDGF-B protects neurons by suppressing the NMDA-evoked current and translocating the glutamate transporter to the cell membrane. The balance between the expression of NMDAR and PDGF-B partly determines the ontogenic susceptibility to brain injury. Enhancement of the PDGF-B/receptor signal pathway may rescue neonatal brains at risk of hypoxic-ischemic injury (19).

These results provide strong evidence for the ability of EPO to repair injured tissue and recover neurological function via increased expression of PDGF-B. The results also provide the first evidence for the involvement of PDGF-B in the neuroprotective effect of EPO on SCI. However, side effects of EPO and the underlying mechanism of its neuroprotective function still require further investigation.

\section{Acknowledgements}

This project was supported by the Health Bureau of Zhejiang Province, China (no. 2009A222).

\section{References}

1. Schmitt C, Miranpuri GS, Dhodda VK, Isaacson J, Vemuganti R and Resnick DK: Changes in spinal cord injury-induced gene expression in rat are strain-dependent. Spine J 6: 113-119, 2006.

2. Hong Z, Chen H, Hong H, Lin L and Wang Z: TSP-1 expression changes in diabetic rats with spinal cord injury. Neurol Res 31: 878-882, 2009

3. Grasso G, Sfacteria A, Meli F, Fodale V, Buemi M and Iacopino DG: Neuroprotection by erythropoietin administration after experimental traumatic brain injury. Brain Res 1182: 99-105, 2007. 
4. Hasselblatt M, Ehrenreich H and Siren AL: The brain erythropoietin system and its potential for therapeutic exploitation in brain disease. J Neurosurg Anesthesiol 18: 132-138, 2006.

5. Ishii Y, Oya T, Zheng L, et al: Mouse brains deficient in neurona PDGF receptor-beta develop normally but are vulnerable to injury. J Neurochem 98: 588-600, 2006.

6. Bergsten E, Uutela M, Li X, et al: PDGF-D is a specific, proteaseactivated ligand for the PDGF beta-receptor. Nat Cell Biol 3 512-516, 2001.

7. Wu QH, Chen WS, Chen QX, Wang JH and Zhang XM: Changes in the expression of platelet-derived growth factor in astrocytes in diabetic rats with spinal cord injury. Chin Med J (Engl) 123: $1577-1581,2010$.

8. Janmaat ML, Heerkens JL, de Bruin AM, Klous A, de Waard V and de Vries CJ: Erythropoietin accelerates smooth muscle cell-rich vascular lesion formation in mice through endothelial cell activation involving enhanced PDGF-BB release. Blood 115 1453-1460, 2010.

9. Montero M, Poulsen FR, Noraberg J, et al: Comparison of neuroprotective effects of erythropoietin (EPO) and carbamylerythropoietin (CEPO) against ischemia-like oxygen-glucose deprivation (OGD) and NMDA excitotoxicity in mouse hippocampal slice cultures. Exp Neurol 204: 106-117, 2007.

10. Okutan O, Solaroglu I, Beskonakli E and Taskin Y: Recombinant human erythropoietin decreases myeloperoxidase and caspase-3 activity and improves early functional results after spinal cord injury in rats. J Clin Neurosci 14: 364-368, 2007.

11. Grasso G, Sfacteria A, Passalacqua M, et al: Erythropoietin and erythropoietin receptor expression after experimental spinal cord injury encourages therapy by exogenous erythropoietin. Neurosurgery 56: 821-827, 2005.
12. Adamcio B, Sargin D, Stradomska A, et al: Erythropoietin enhances hippocampal long-term potentiation and memory. BMC Biol 6: 37, 2008.

13. King VR, Averill SA, Hewazy D, Priestley JV, Torup L and Michael-Titus AT: Erythropoietin and carbamylated erythropoietin are neuroprotective following spinal cord hemisection in the rat. Eur J Neurosci 26: 90-100, 2007.

14. Bittorf T, Buchse T, Sasse T, Jaster R and Brock J: Activation of the transcription factor NF-kappaB by the erythropoietin receptor: structural requirements and biological significance. Cell Signal 13: 673-681, 2001.

15. Zhang W, Sun B, Wang X, Liu J, Zhang Z and Geng S: Erythropoietin enhances survival of facial motor neurons by inhibiting expression of inducible nitric oxide synthase after axotomy. J Clin Neurosci 17: 368-371, 2010.

16. Enge M, Wilhelmsson U, Abramsson A, et al: Neuron-specific ablation of PDGF-B is compatible with normal central nervous system development and astroglial response to injury. Neurochem Res 28: 271-279, 2003

17. Yang C, Li B, Liu TS, Zhao DM and Hu FA: Effect of electracupuncture on proliferation of astrocytes after spinal cord injury. Zhongguo Zhen Jiu 25: 569-572, 2005 (In Chinese).

18. Huang X, Kim JM, Kong TH, et al: GM-CSF inhibits glial scar formation and shows long-term protective effect after spinal cord injury. J Neurol Sci 277: 87-97, 2009.

19. Egawa-Tsuzuki T, Ohno M, Tanaka N, et al: The PDGF B-chain is involved in the ontogenic susceptibility of the developing rat brain to NMDA toxicity. Exp Neurol 186: 89-98, 2004. 